1991-03-01

\title{
Identifiability in wind estimation from scatterometer measurements
}

David G. Long

david_long@byu.edu

Jerry M. Mendel

Follow this and additional works at: https://scholarsarchive.byu.edu/facpub

Part of the Electrical and Computer Engineering Commons

\section{Original Publication Citation}

Long, D. G., and J. M. Mendel. "Identifiability in Wind Estimation from Scatterometer

Measurements." Geoscience and Remote Sensing, IEEE Transactions on 29.2 (1991): 268-76

\section{BYU ScholarsArchive Citation}

Long, David G. and Mendel, Jerry M., "Identifiability in wind estimation from scatterometer measurements" (1991). Faculty Publications. 723.

https://scholarsarchive.byu.edu/facpub/723

This Peer-Reviewed Article is brought to you for free and open access by BYU ScholarsArchive. It has been accepted for inclusion in Faculty Publications by an authorized administrator of BYU ScholarsArchive. For more information, please contact ellen_amatangelo@byu.edu. 


\title{
Identifiability in Wind Estimation From Scatterometer Measurements
}

\author{
David G. Long, Member, IEEE, and Jerry M. Mendel, Fellow, IEEE
}

\begin{abstract}
In this paper we consider the problem of identifiability of a wind vector that is estimated from wind scatterometer measurements of the radar backscatter of the ocean's surface. The traditional wind estimation approach produces multiple estimates of the wind direction. A second processing step, known as "dealiasing" or "ambiguity removal" is used to select a single wind estimate from these multiple solutions. Dealiasing is typically based on various ad hoc considerations. The traditional wind estimation approach results in multiple solutions associated with local minima in an objective function formed from the noisy backscatter measurements. We address the question of the uniqueness of the wind vector estimates resulting from this intuitive approach. We show that wind vector estimation using scatterometer measurements is set-wise or system identifiable; i.e., we provide mathematical proof that there is a unique set of wind vectors that could have given rise to the observed backscatter values, and that the set of wind vector estimates is consistent. The fact that the wind vector estimate is a set arises from the nature of the relationship between the backscatter and wind vector. Within this set there is no way to select a unique wind vector estimate from the measurements at a single sample point; hence, we establish a theoretical reason for dealiasing.
\end{abstract}

\section{INTRODUCTION}

A WIND scatterometer is an active radar remote sensing instrument which provides measurements of the normalized backscatter $\left(\sigma^{\circ}\right)$ of the ocean's surface [19]. From these noisy measurements of $\sigma^{\circ}$, the speed and direction of the wind over the ocean's surface may be inferred using an empirically derived relationship between the wind vector and $\sigma^{\circ}$ known as the "geophysical model function" [13]-[15], [20].

In the traditional wind estimation approach, an objective function (typically based on maximum likelihood or least squares) is formulated using the $\sigma^{\circ}$ measurements and is minimized to obtain estimates of the wind vector. Due to the nature of the geophysical model function the objective function has several local minima. The wind vectors associated with each of these local minima are termed "aliases" or "ambiguities" [21]. Traditionally, all the aliases are retained in a set which we call the "noisy ambiguity set" from which later processing, known as "de-aliasing" or "ambiguity removal" (see [16], [17], [21]), is used to select a single wind-vector estimate.

While this process works reasonably well in practice (see, for example, [19] and [6]), there are significant difficulties with the method [15], [21]. As yet, a theoretical basis for this approach is lacking; no one has shown that a consistent estimate of the surface wind is uniquely determined using this two-step procedure. We provide such a basis by showing that for the first

Manuscript received October 20, 1989; revised August 6, 1990.

D. G. Long is with the Department of Electrical and Computer Engineering, 459 CB, Brigham Young University, Provo, UT 84602.

J. M. Mendel is with the Signal and Image Processing Institute, Department of Electrical Engineering-Systems, University of Southern California, Los Angeles, CA 90089.

IEEE Log Number 9041436 step the surface wind vector (or, equivalently, the wind speed and direction) is identifiable from the wind scatterometer measurements.

"Identifiability" is an important concept central to estimation theory. Loosely speaking, identifiability indicates whether or not an estimation procedure will yield a unique and consistent estimate of the desired parameters from the available measurements [4], [8], [12], [18]. A more formal definition of identifiability will be given in Section IV. Excellent discussions of identifiability as applied to linear systems are given by Ljung [8] and Soderstrom and Stoica [18]

In this paper we consider the problem of identifiability of the surface wind vector from wind scatterometer measurements at a given observation point. We will show that, whereas the wind vector estimate is not unique, it is set-wise or system identifiable; i.e., there is a unique set of wind vectors corresponding to the observed $\sigma^{\circ}$ values which cannot be distinguished from one another using just the $\sigma^{\circ}$ measurements. These results provide theoretical credence to the historical practice of using the noisy ambiguity sets from point-wise wind estimation and dealiasing. Identifiability for the second dealiasing step is not addressed, since dealiasing relies on information not present in the $\sigma^{\circ}$ measurements.

In this paper we first discuss the conditions (which are due to the nature of the geophysical model function) under which more than one wind vector can give rise to the same true noise-free measurements of $\sigma^{\circ}$. We call the set of wind vectors which give rise to the same noise-free values of $\sigma^{\circ}$ the "true ambiguity set," since it is not possible to uniquely select a wind vector from this set. Selection of a single wind estimate must be done using other considerations; hence the need for dealiasing. We then discuss the wind scatterometer measurement model, provide a formal definition and discussion of the significance of identifiability, and provide an outline of the proof of identifiability of wind vectors from scatterometer measurements.

\section{The Relationship Between $\sigma^{\circ}$ And the Vector WIND}

A wind scatterometer does not directly measure $\sigma^{\circ}$, but infers $\sigma^{\circ}$ from noisy measurements of the backscattered power of an RF pulse directed at the ocean's surface. We defer further discussion of the power measurement process until the next section. $\sigma^{\circ}$ is related to the wind vector over the ocean's surface at the observation point by a nonlinear geophysical model function which we denote by $\mathfrak{K} . \sigma^{\circ}$ is a function of the relative azimuth angle between the radar illumination and wind direction $(\chi)$, the wind speed $(U)$, the incidence angle of the radar illumination $(\theta)$, and the radar polarization $(p)$ [19]. The relationship between the wind and $\sigma^{\circ}$ has been extensively studied in the literature (e.g., [13]-[15], [20]). For this paper we 


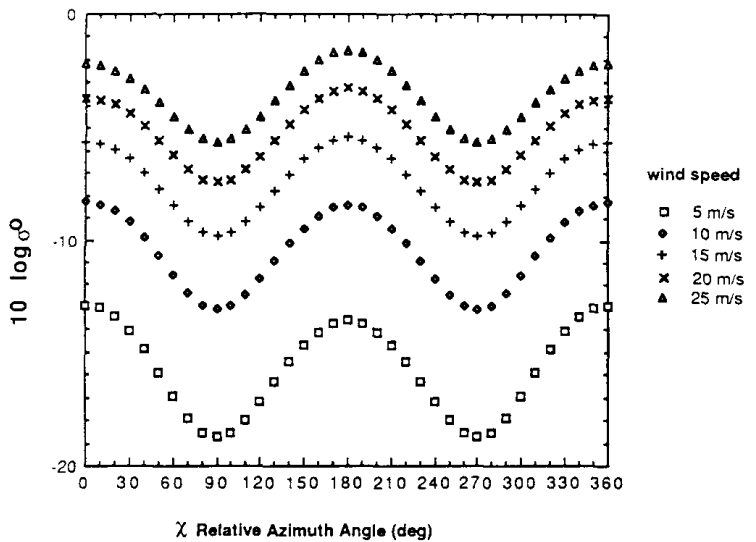

Fig. 1. $\sigma^{\circ}$ versus $\chi$ for several wind speeds for an incidence angle of $30^{\circ}$; vertical polarization using the SASS $^{1}$ model function was used.

will assume that the geophysical model function is known and ignore any geophysical modeling error. Fig. 1 illustrates the relationship between $\sigma^{\circ}, \chi$, and $U$ for the well-known SASS ${ }^{1}$ model function at $14.6 \mathrm{GHz}[15]$. Note the $\cos 2 \chi$ dependence of $\sigma^{\circ}$ on the wind direction. While other model functions (e.g., [13], [20]) differ in detail, they share a similar $\cos 2 \chi$ dependence on wind direction. This characteristic of the model function is primarily responsible for the difficulties in obtaining unique estimates of the wind vector from $\sigma^{\circ}$ measurements.

Since $\mathfrak{M}$ has a multivalued inverse, several measurements of $\sigma^{\circ}$ from different azimuth angles must be used to estimate the wind vector. The Seasat scatterometer (SASS) provided measurements of $\sigma^{\circ}$ at two different azimuth angles separated by approximately $90^{\circ}[6]$. The planned NSCAT scatterometer will provide measurements of $\sigma^{\circ}$ at three different azimuth angles separated by $65^{\circ}$ and $90^{\circ}$, respectively, from the first azimuth observation angle [7]. For a given observation point, let $\sigma^{\circ}(k)$ be the true value of $\sigma^{\circ}$ corresponding to the $k$ th observation. For notational simplicity, we write,

$$
\sigma^{\circ}(k)=\mathfrak{M}\{(u, v), k\}
$$

where $u$ and $v$ are the components of the wind vector at the observation point, and where the dependence of $\sigma^{\circ}$ on the observation azimuth angle, the incidence angle, and radar polarization are subsumed in the index $k$ of $\mathfrak{T}$.

Due to the nature of $\mathfrak{M}$ and the fact that $\sigma^{\circ}$ is observed from only a small set of azimuth angles, there may be several wind vectors which give rise to the same set of $\sigma^{\circ}$ values (one value for each observation azimuth angle). Define $D^{c}$ to be the set of all possible true wind velocity vectors, denoted by $\left(u_{t}, v_{t}\right)$, which give rise to the same set of $\sigma^{\circ}(k)$ 's; i.e.,

$$
D^{c} \triangleq\left\{(u, v) \mid \mathfrak{N}\{(u, v), k\}=\mathfrak{N}\left\{\left(u_{t}, v_{t}\right), k\right\} \forall k\right\} .
$$

Note that by definition, $\left(u_{t}, v_{t}\right) \in D^{c}$.

Because members of the set $D^{c}$ produce exactly the same set of $\sigma^{\circ}(k)$ values, they cannot be distinguished from one another, even if the measurements of $\sigma^{\circ}$ are noise free. We refer to $D^{c}$ as the "true ambiguity set." The membership in the set $D^{c}$ depends on the model function, the set of relative azimuth angles (and the corresponding incidence angles and polarizations of the antenna beams), and the true wind vector. Depend- ing on these factors, $D^{c}$ will contain one or more members. The fact that $D^{c}$ may contain multiple members is a property inherent to the geophysical model function and the measurement geometry.

In effect, the geophysical model function creates a many-toone mapping of wind velocity vectors to the set of measurement $\sigma^{\circ}(k)$ 's corresponding to the observation azimuth angles. The best we can ever expect to do is identify all members of $D^{c}$. Selection of a unique wind vector from $D^{c}$ requires additional information not contained in the $\sigma^{\circ}$ measurements for a single sample point of the ocean's surface; hence the need for dealiasing in which data from other sample points (or from other sources) are used in conjunction with dynamical constraints, continuity considerations, etc., to select a wind vector field which (hopefully) is "close" to the true field [16], [17], [21].

To illustrate the membership of $D^{c}$ for various true wind vectors, let us consider the well-known SASS ${ }^{1}$ model function [15], for which $\mathfrak{M}$ is expressed as

$\mathfrak{M}\{(u, v), k\}=G(\theta(k), \chi(k), p(k)) U^{H(\theta(k), \chi(k), p(k))}$

where $U=|(u, v)|$ is the wind speed, $\chi(k)=\psi(k)-\phi$, where $\phi=\tan ^{-1}(v / u)$ is the wind direction, $\psi(k)$ is the azimuth angle of the $k$ th antenna beam, and $p(k)$ is the radar polarization. $\chi$ is known as the relative azimuth angle. $G$ and $H$ are tabular functions. For given values of $\theta$ and $p, G(\theta, \chi, p)$ $\sim \cos 2 \chi$ and $H(\theta, \chi, p) \sim 2$ [15]. For given values of $\sigma^{\circ}(k)$, $\chi(k), \theta(k)$, and $p(k), U$ can be computed as

$$
U=\exp \left\{\frac{1}{H(\theta(k), \chi(k), p(k))} \ln \left[\frac{\sigma^{\circ}(k)}{G(\theta(k), \chi(k), p(k))}\right]\right\} .
$$

Consider the SASS antenna configuration with two azimuth angles (both vertically polarized in this example) separated by $90^{\circ}$. For a single true wind vector $\left(u_{t}, v_{t}\right)$, the set of true $\sigma^{\circ}$ values are denoted $\sigma^{\circ}(k=1)=\mathfrak{M}\left\{\left(u_{t}, v_{t}\right), k=1\right\}$ and $\sigma^{\circ}(k$ $=2)=\mathfrak{T}\left\{\left(u_{t}, v_{t}\right), k=2\right\}$. Now consider the two curves in wind vector space $(u, v)$, defined by $\mathfrak{T}\{(u, v), 1\}=\sigma^{\circ}(1)$ and $\mathfrak{T}\{(u, v), 2\}=\sigma^{\circ}(2)$. These curves are depicted in Fig. 2(a) for a true wind speed of $3 \mathrm{~m} / \mathrm{s}$ and a true wind direction of $0^{\circ}$, where the wind vector $(u, v)$ has been converted to speed and direction with the direction angle relative to the antenna azimuth angle used in plotting. $k=1$ corresponds to the relative observation azimuth angle of $0^{\circ}$, while $k=2$ corresponds to $90^{\circ}$. For this example an incidence angle of $40^{\circ}$ was used for both measurements. To generate this curve for $k=1$, given $\sigma^{\circ}(1)$ and the respective $\psi(1), \theta(1)$, and $p(1)$ values for each possible value of $\phi$ (and, subsequently, $\chi$ ), a wind speed $U$ was computed using (4) such that $\mathfrak{T}\{(U \cos \chi(1), U \sin \chi(1))$, $1\}=\sigma^{\circ}(1)$. The curve for $k=2$ was similarly computed. These curves intersect several times. The points along these curves at which they intersect define the true ambiguity set $D^{c}$. Curves for other true wind directions are also shown in Fig. 2. Note that depending on the wind direction, the number of elements in $D^{c}$ varies from 1 to 4 .

Fig. 3 shows maps of $D^{c}$ for a true wind speed of $3 \mathrm{~m} / \mathrm{s}$ at two different incidence angles. To prepare Fig. 3(a), a true wind direction (along the horizontal axis) was first selected, and $D^{c}$ for that true wind direction was computed. The wind direction of each of the elements of $D^{c}$ (the directions of each the elements of the "true ambiguity set") was then marked along the 


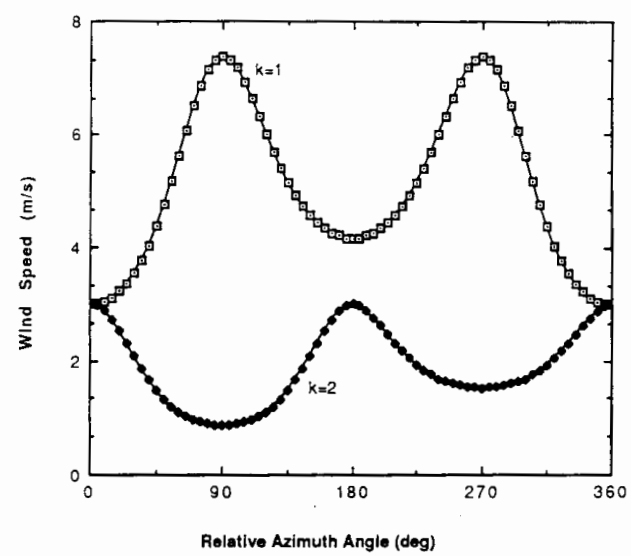

(a)

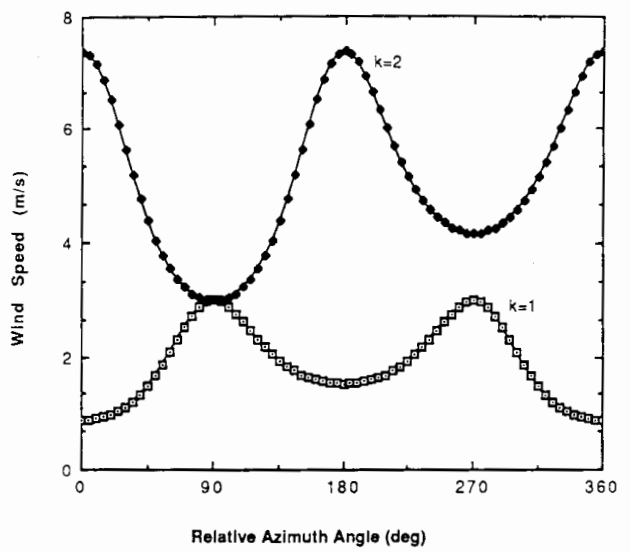

(c)

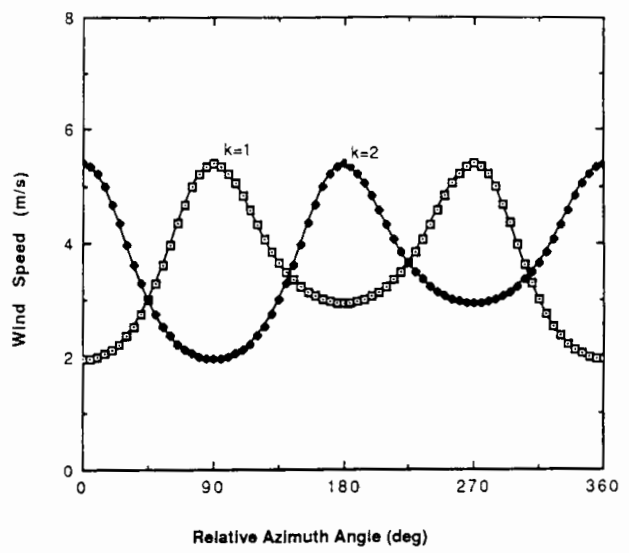

(b)

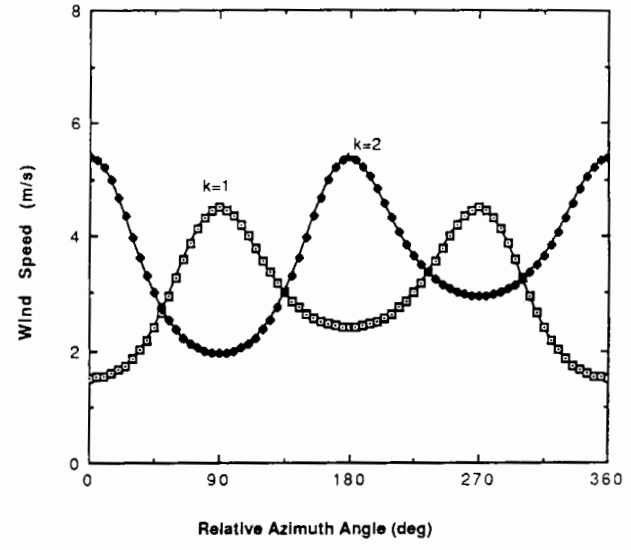

(d)

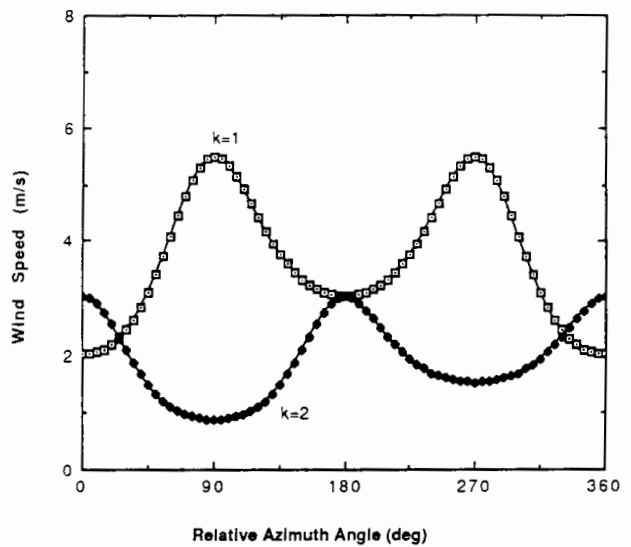

(e)

Fig. 2. The curves in $(u, v)$ space, along which $\mathfrak{M}\{(u, v), k\}=\mathfrak{M}\left\{\left(u_{t}, v_{t}\right), k\right\}$ for $k=1,2 .\left(u_{t}, v_{t}\right)$ is the true wind speed of $3 \mathrm{~m} / \mathrm{s}$ and azimuth angle (relative to the first antenna beam) of: (a) $0^{\circ}$; (b) $45^{\circ}$; (c) $90^{\circ}$; (d) $135^{\circ}$; and (e) $180^{\circ}$, where the observation azimuth angles are $0^{\circ}$ and $90^{\circ}$ for $k=1$ and $k=2$, respectively. Both antennas were vertically polarized and had an incidence angle of $40^{\circ}$. The SASS ${ }^{1}$ model function was used to generate these plots.

vertical corresponding to the true wind direction. This process was repeated for each true wind direction. The result graphically illustrates how $D^{c}$ changes with the true wind direction for the SASS ${ }^{1}$ model function. The vertical lines labeled (a)-(e) correspond to the plots shown in Fig. 2. Fig. 3(b) was generated in a similar manner, but for a different incidence angle of $20^{\circ}$. 


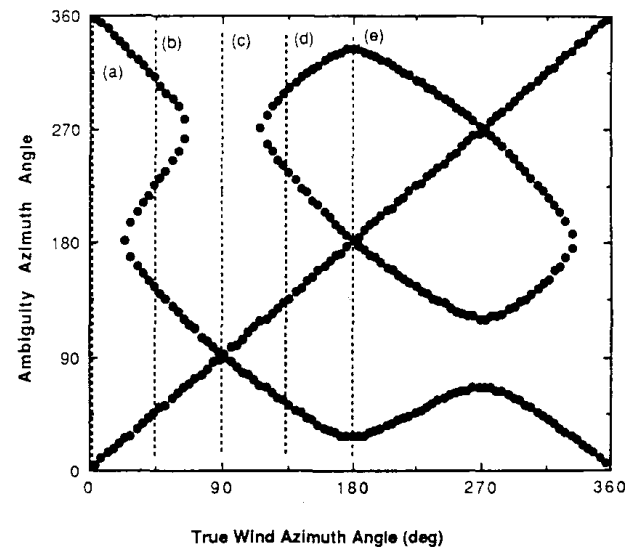

(a)

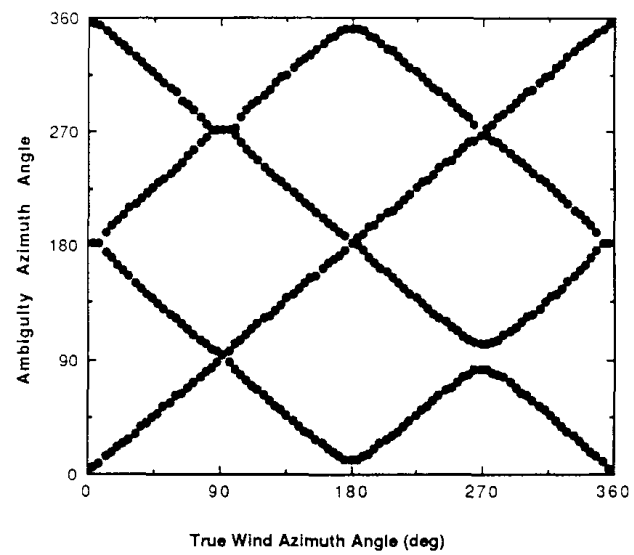

(b)

Fig. 3. A map of the true ambiguity set for a true wind speed of $3 \mathrm{~m} / \mathrm{s}$ at an incidence angle of: (a) $40^{\circ}$; and (b) $20^{\circ}$. The labeled vertical lines on (a) correspond to the results from the plots shown in Fig. 2. Two observation azimuth angles at $0^{\circ}$ and $90^{\circ}$ (SASS configuration), both vertically polarized, were used with the SASS ${ }^{1}$ model function (see text).

For purposes of comparison, Fig. 4 was prepared in the same manner as Fig. 3, but for the three-azimuth-angle NSCAT scatterometer configuration with all vertically polarized antennas. Note that the additional azimuth angle reduces the number of intersections at a given true wind direction to 1 or 2 .

Figs. 3 and 4 can be misleading, since they show the true ambiguity set. The true ambiguity set can only be obtained from noise-free $\sigma^{\circ}$ measurements. In the real world, the $\sigma^{\circ}$ measurements are always noisy. When the $\sigma^{\circ}$ measurements are noisy additional intersections may be produced or the curves may not intersect at all. When the measurements are noisy, an objective function (such as the maximum-likelihood (ML) objective function given in Section V) is formulated from the measurements and minimized to provide estimates of the wind vector [3]. In the noisy case, the wind vectors corresponding to the local minima of the objective function define the noisy ambiguity set. The number of minima depends on the true wind, the observa tion geometry, measurement integration time, and the noise realization. To contrast the difference between the true and noisy ambiguity sets, Figs. 5 and 6 were prepared, with Fig. 5 cor-

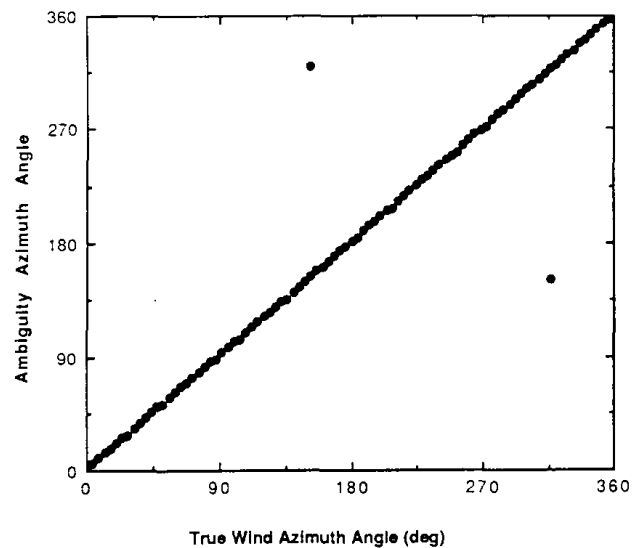

(a)

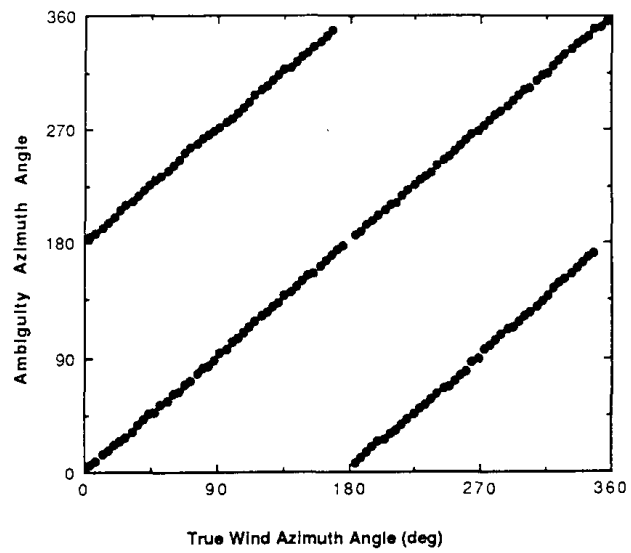

(b)

Fig. 4. Same as Fig. 3, but for the three observation azimuth angles $\left(0^{\circ}\right.$ $65^{\circ}$, and $90^{\circ}$ ) NSCAT configuration (all vertically polarized)

responding to the SASS antenna configuration, and Fig. 6 to the NSCAT configuration. Fig. 5 should be compared with Fig. 3, whereas Fig. 6 should be compared with Fig. 4. Figs. 5 and 6 were prepared as follows: For a given true wind speed and direction the true $\sigma^{\circ}$ set was computed. Noisy $\sigma^{\circ}$ measurements were simulated by Monte Carlo methods, and the noisy ambiguity set corresponding to each realization of the noisy $\sigma^{\circ}$ sets was determined using the ML objective function [3]. While details vary with the choice of objective function (see [3]), the general characteristics remain the same. A total of 500 realizations for each true wind direction was used. A histogram of the directions of the elements of the noisy ambiguity sets was computed. This was repeated for each true direction and the resulting histograms plotted. For a given true wind direction, the average number and location of the wind directions corresponding to the noisy ambiguity set can be seen. Note that there are typically 2 to 4 members of the noisy ambiguity set, regardless of the number of members of the true ambiguity set.

The increased number of elements of the noisy ambiguity set relative to the true ambiguity set is due to the presence of noise. The difference between the noisy ambiguity set and true ambiguity set is, in fact, one of the reasons that identifiability of the 


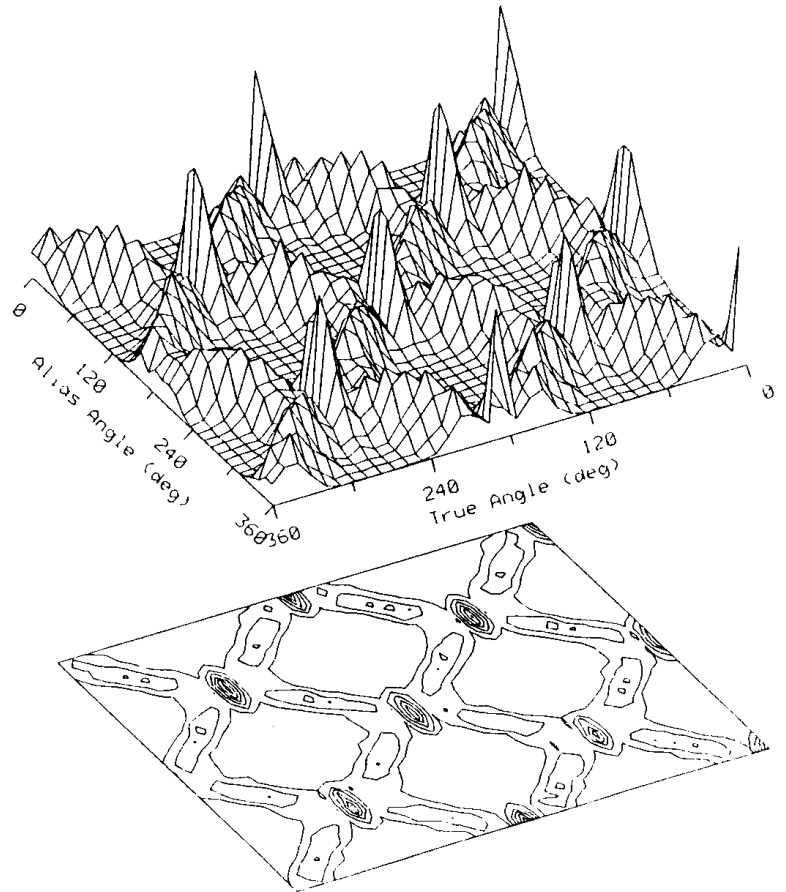

(a)

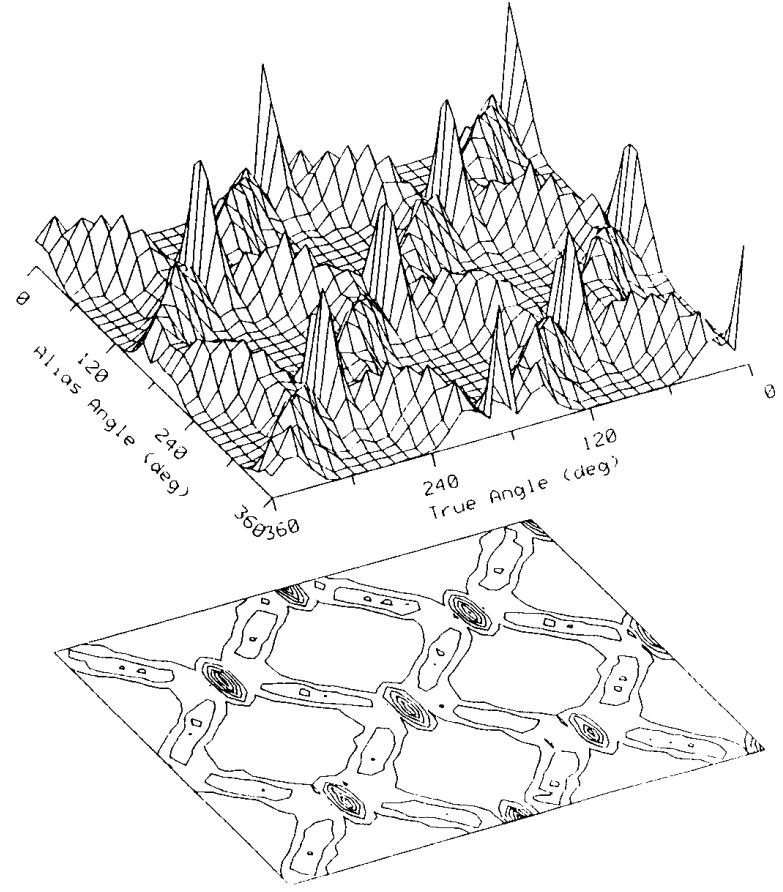

(b)

Fig. 5. Histogram of the directions of the noisy ambiguity sets versus true wind direction. The histograms were created by Monte Carlo simulations of noisy $\sigma^{\circ}$ measurements using the SOS objective function [3]. The true wind speed is $3 \mathrm{~m} / \mathrm{s}$. The incidence angle is: (a) $40^{\circ}$, and (b) $20^{\circ}$ for both vertically polarized antenna beams in the SASS configuration. The SASS model function was used (see caption of Fig. 3). Contours are arbitrary.

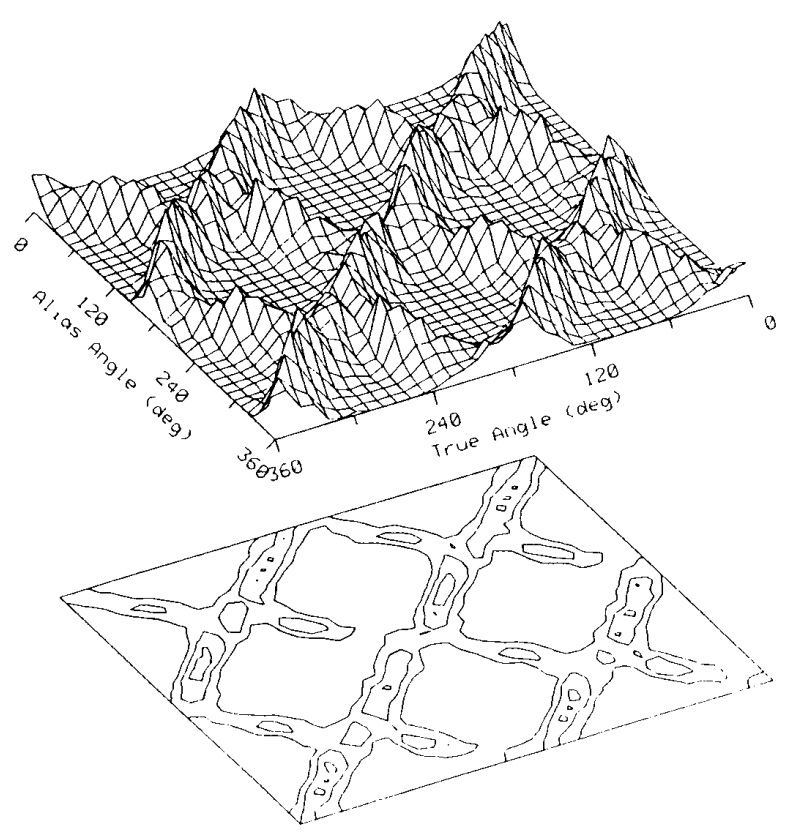

(a)

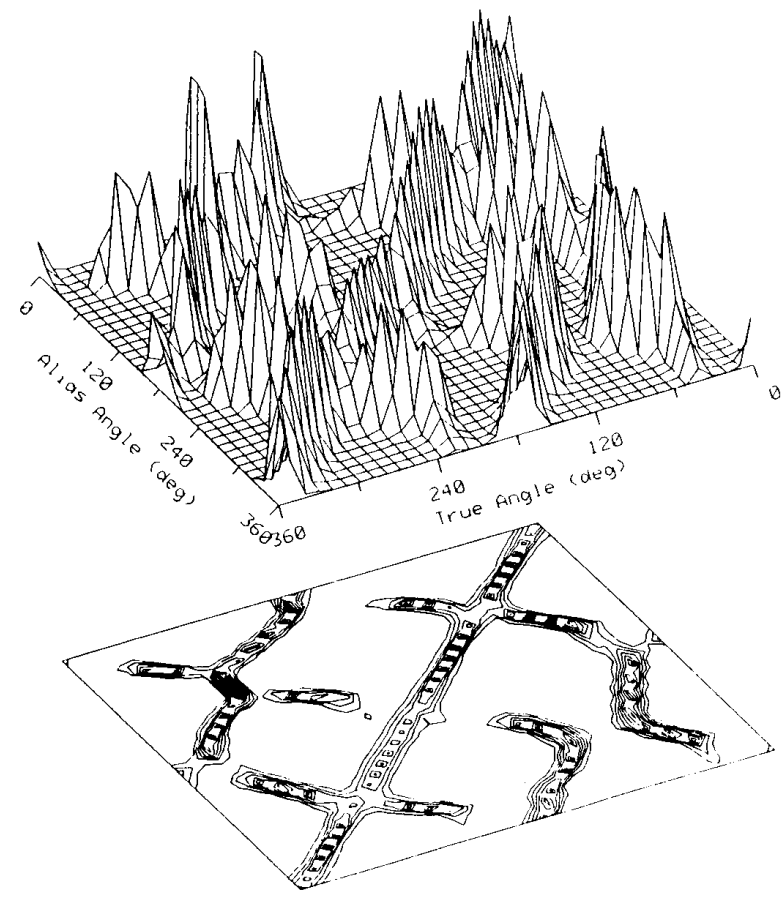

(b)

Fig. 6. Same as Fig. 5, but for the NSCAT configuration with all vertically polarized beams. 


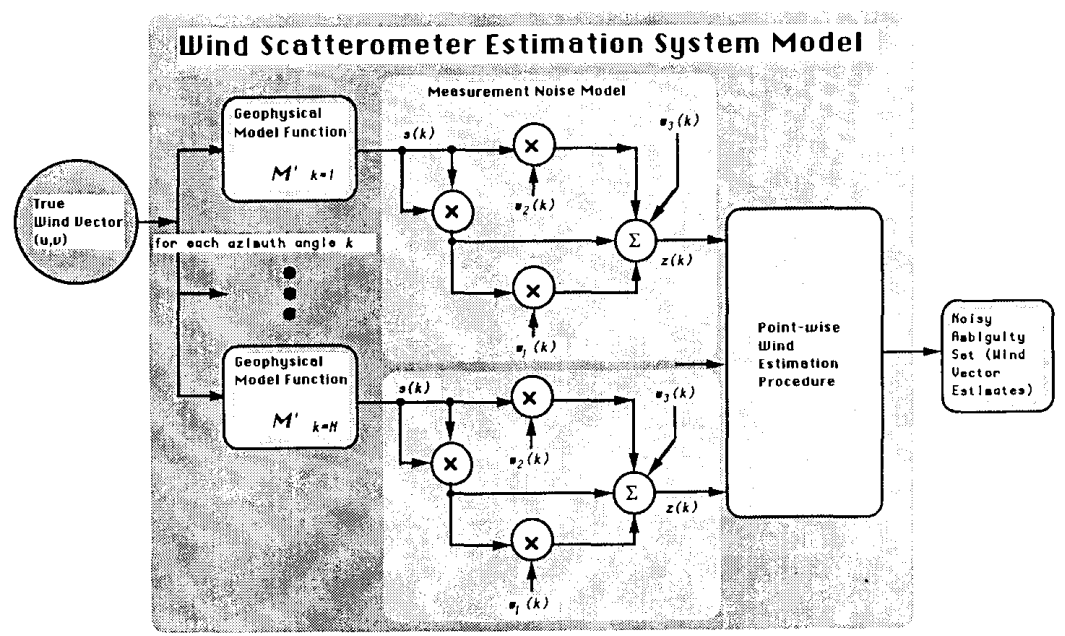

Fig. 7. Diagram of the wind scatterometer measurement model. Note that the parameter $s(k)$ is the square root of $\sigma^{\circ}(k)$ (see text). $w_{1}(k), w_{2}(k)$, and $w_{3}(k)$ are independent, zero-mean Gaussian random variables with variances which depend on $k$ Geophysical modeling error in $\mathfrak{T}^{\prime}$ is not considered in this system model.

wind vector estimation has been questioned. We will show that, as the measurement noise level is reduced (by increasing the measurement time), the noisy ambiguity set (corresponding to the ML objective function) converges to the true, multimembered ambiguity set $D^{c}$; i.e., that wind vector estimation is system identifiable. Even if we could choose the observation angles to ensure that the true ambiguity set $D^{c}$ contains a single member for all true winds, the noisy ambiguity set will generally be multimembered. In either case, if a unique wind estimate is desired, dealiasing is required.

\section{The Scatterometer Measurement Process}

In this section we review the scatterometer measurement process and discuss the noise model used in our identifiability proof. Additional information can be found in [11] and [19].

Let us consider the measurement process for a particular observation $k$ at a particular sample point within the measurement swath. The radar scatterometer transmits a radar pulse of known energy towards the ocean's surface. A portion of this power is reflected, or backscattered, toward the radar. The amount of power in the return echo (signal) is measured by the scatterometer. The power received is a function of the known radar equation parameters and $\sigma^{\circ}$, which is a function of the wind over the ocean's surface. The measurement of the backscattered power is corrupted by additive thermal noise. A separate measurement of the noise-only power is made and subtracted from the signal plus noise power measurement to estimate the signal power. The $\sigma^{\circ}$ measurement is obtained from the signal power measurement using the radar equation [1], [19]. We denote the resulting $\sigma^{\circ}$ measurement made at the $k$ th azimuth angle $z(k)$. Consequently, at each sample point a noisy measurement $z(k)$ of the true $\sigma^{\circ}$, denoted $\sigma^{\circ}(k)$, is obtained for each of $k=1$, $\cdots, N(N=2$ or 3$)$ azimuth angles. The model for the noisy measurement $z(k)$ of the true $\sigma^{\circ}$ value is

$$
z(k)=\sigma^{\circ}(k)+\nu(k)
$$

where $\nu(k)$ is a zero-mean Gaussian random variable whose variance is dependent on the true $\sigma^{\circ}, \sigma^{\circ}(k)$, and the time length $T$ of the measurement [2], [19].

The variance of the signal power measurement (and hence the $\sigma^{\circ}$ measurement) is a quadratic function of $\sigma^{\circ}(k)$ [2], [5]. The variance of $z(k)$ can be expressed as [2], [3], [5]

$$
\operatorname{Var}[z(k)]=\alpha^{2}(k) \sigma^{\circ 2}(k)+\beta^{2}(k) \sigma^{\circ}(k)+\gamma^{2}(k)
$$

where $\alpha(k), \beta(k)$, and $\gamma(k)$ depend on the known observation azimuth angle and the length $T$ of the return echo integration period. $\alpha(k), \beta(k)$, and $\gamma(k)$ are all proportional to $1 / \sqrt{T}$.

To simplify the notation in the remainder of the paper, we define $s(k)$ as

$s(k) \triangleq \sqrt{\sigma^{\circ}(k)}=\mathfrak{M}^{\prime}\{(u, v), k\}=\sqrt{\mathfrak{M}\{(u, v), k\}}$.

Using this definition, we can write the general noise model for $\sigma^{\circ}(k)$ in terms of the modified measurement parameter $s(k)$ (by combining (5) and (6)) as

$$
z(k)=s^{2}(k)\left[1+w_{1}(k)\right]+s(k) w_{2}(k)+w_{3}(k)
$$

where $w_{1}(k), w_{2}(k)$, and $w_{3}(k)$ are independent, zero-mean Gaussian random variables with known variances $\alpha^{2}(k), \beta^{2}(k)$, and $\gamma^{2}(k)$, respectively. A diagram of the resulting scatterometer measurement noise model is shown in Fig. 7.

In Section V, we make use of the conditional probability distribution of $z(k)$ given $s(k)$; it is given as

$$
\begin{aligned}
p(z(k) \mid s(k)) & \\
= & \frac{1}{\sqrt{2 \pi}} \frac{1}{\left[\alpha^{2}(k) s^{4}(k)+\beta^{2}(k) s^{2}(k)+\gamma^{2}(k)\right]^{1 / 2}} . \\
& \cdot \exp \left\{-\frac{1}{2}\left[z(k)-s^{2}(k)\right]^{2} /\left[\alpha^{2}(k) s^{4}(k)\right.\right. \\
& \left.\left.+\beta^{2}(k) s^{2}(k)+\gamma^{2}(k)\right]\right\} .
\end{aligned}
$$


Measurement $z(k)$ is a random variable due to the presence of the noise $\nu(k)$. Since $\alpha^{2}(k), \beta^{2}(k)$, and $\gamma^{2}(k)$ can be expressed as

$$
\begin{aligned}
\alpha^{2}(k) & =\frac{1}{T} a(k) \\
\beta^{2}(k) & =\frac{1}{T} b(k) \\
\gamma^{2}(k) & =\frac{1}{T} c(k)
\end{aligned}
$$

where $a(k), b(k)$, and $c(k)$ are positive constants that are independent of the measurement time $T$, we see that as $T \rightarrow \infty$, $\alpha^{2}(k) \rightarrow 0, \beta^{2}(k) \rightarrow 0$, and $\gamma^{2}(k) \rightarrow 0$. This corresponds to reducing the measurement noise to zero, which is equivalent to reducing the so-called measurement $K_{p}$ (see [3], [5]) to zero. Note that $K_{p}$ can also be decreased by increasing the measurement signal-to-noise ratio. From (6) and (10) it follows that Var $[z(k)] \rightarrow 0$ as $T \rightarrow \infty$; hence $z(k)$ converges in probability to the deterministic quantity $s^{2}(k)=\sigma^{\circ}(k)$. This result is used in the identifiability proof.

\section{IDENTIFIABILITY}

In this section we discuss the meaning and significance of identifiability. In order to give a formal definition of identifiability, define $\hat{\theta}(T)$ to be an estimate of the desired parameter vector $\theta$ (in this case, the wind vector). $\hat{\theta}(T)$ is a function of the measurements (which depend on the time $T$ used to make the measurements), the true system (including the true wind vector), the estimation scheme, and the system model. The set $D^{c}$ is the set of parameter vectors for which the system model gives a perfect description of the true system. When $D^{c}$ consists of more than one point, there will be several parameter vectors within the system model which will give a perfect description of the true system. Then the system may be said to be "system identifiable" for a given estimation scheme if [18]

$$
\lim _{T \rightarrow \infty} \inf _{\theta \in D^{c}}\|\hat{\theta}(T)-\theta\|=0 .
$$

While the parameter estimate may not be unique, the estimate will be consistent to within the unique set $D^{c}$. If $D^{c}$ consists of only a single point, the parameter estimate will be both consistent and unique.

For scatterometer wind estimation the system model includes: (i) The scatterometer measurement geometry (including the number, the polarization, and the azimuth and incidence angles of the measurements); (ii) the geophysical model function $\mathfrak{N}$ relating $\sigma^{\circ}$ and the wind; and (iii) the $\sigma^{\circ}$ noise measurement model (described in Section III). We assume that the geometric quantities and $\mathfrak{M}$ are known. The estimation approach we employ is the maximum-likelihood.

Our interest in the identifiability of this system arises from two sources: (i) The bi-cosine nature of the geophysical model function which results in multimembered true ambiguity sets; and (ii) the multiplicative nature of the measurement noise. In regard to the latter, consider estimating a signed parameter $\theta$ from measurements $z(k)=\theta n(k)$, where $n(k)$ is the zero-mean Gaussian process. If the variance of $n(k)$ is known, a consistent estimate of the magnitude of $\theta$ can be obtained, but not the sign of $\theta$; hence $\theta$ is not uniquely identifiable. If, however, the vari- ance of $n(k)$ is unknown, it is not possible to formulate a consistent estimate of either the magnitude or the sign of $\theta$, and therefore $\theta$ is not identifiable.

\section{IDENTIFIABILITy of THE SET $D^{c}$}

Let us consider the identifiability of $u$ and $v$ (or, equivalently, the wind speed and direction). As mentioned above, $\left(u_{t}, v_{t}\right)$ is the true wind vector and $D^{c}$ is the corresponding true ambiguity set. Let $Z_{p}=(z(1), \cdots, z(N))^{T}$; its elements are statistically independent. From (9), the conditional probability of $Z_{p}$ given $(u, v)$ is

$$
\begin{aligned}
p\left(Z_{p} \mid u, v\right) & \\
= & \prod_{k=1}^{N} \frac{1}{\sqrt{2 \pi}} \frac{1}{\left[\alpha^{2}(k) s^{4}(k)+\beta^{2}(k) s^{2}(k)+\gamma^{2}(k)\right]^{1 / 2}} \\
& \cdot \exp \left\{-\frac{1}{2}\left[z(k)-s^{2}(k)\right]^{2} /\left[\alpha^{2}(k) s^{4}(k)\right.\right. \\
& \left.\left.+\beta^{2}(k) s^{2}(k)+\gamma^{2}(k)\right]\right\} .
\end{aligned}
$$

The log-likelihood function [12] $L(u, v)$ is given as

$$
\begin{aligned}
L(u, v)= & \sum_{k=1}^{N}\left\{-\frac{1}{2} \log \left[\alpha^{2}(k) s^{4}(k)+\beta^{2}(k) s^{2}(k)+\gamma^{2}(k)\right]\right. \\
& -\frac{1}{2}\left[z(k)-s^{2}(k)\right]^{2} /\left[\alpha^{2}(k) s^{4}(k)\right. \\
& \left.\left.+\beta^{2}(k) s^{2}(k)+\gamma^{2}(k)\right]\right\}
\end{aligned}
$$

The maximum-likelihood objective function is the negative of $L(u, v)$. The ML estimates are obtained by minimizing the ML objective function.

To show identifiability of the point-wise estimation scheme using the ML objective function, we need to show that as $T \rightarrow$ $\infty$ (corresponding to a longer and longer measurement (for which the noise variance goes to zero)), the locations of global minima of $-L(u, v)$ converge in probability to the members of $D^{c}$ [4], [8].

To show convergence in probability we first show that: (a) $-L(u, v) / T$ converges in the mean-squared sense (which is stronger than convergence in probability) to the deterministic function $E[-L(u, v) / T]$; and (b) that the set of maximumlikelihood estimates of $u$ and $v$ converge in probability to the location of the minimum of $E[-L(u, v) / T]$. Doing this is equivalent to showing that the set $A_{p}^{c}$ of the $(u, v)$ which minimize $-L(u, v) / T$ for $T \rightarrow \infty$ is equal to $D^{c}$, where $A_{p}^{c}$ is defined as $[4],[8]$

$$
\begin{aligned}
A_{p}^{c} & =\left\{\left(u_{1}, v_{1}\right) \mid \lim _{T \rightarrow \infty} E\left[\frac{-L\left(u_{1}, v_{1}\right)}{T}\right]\right. \\
& \left.=\min _{u, v} \lim _{T \rightarrow \infty} E\left[\frac{-L(u, v)}{T}\right]\right\} .
\end{aligned}
$$

We note that the set $A_{p}^{c}$ is the set of maximum-likelihood estimates of $(u, v)$ as $T \rightarrow \infty$. Hence if $A_{p}^{c}=D^{c}$, the maximumlikelihood estimate is consistent and the set $D^{c}$ is identifiable [4].

Since the mathematical details of the proof are tedious, we provide only an outline of the procedure used in the identifi- 
ability proof here; the full details are contained in [11]. The procedural outline is as follows.

To show (a) we first compute the limit of $E[-L(u, v) / T]$ as $T \rightarrow \infty$. Using the results given in Section III, this limit is seen to be both non-negative and deterministic; i.e., it is not a function of the realization of the noise. We then compute $E\left\{[-L(u, v) / T-E[-L(u, v) / T]]^{2}\right\}$. In the limit as $T \rightarrow$ $\infty$, this expression converges to zero, which proves the desired result (a).

To show (b), we note that because convergence in the meansquare sense is stronger than convergence in probability, it follows that since $-L(u, v) / T$ converges in the mean-square to the deterministic function $E[-L(u, v) / T]$ in the limit as $T \rightarrow$ $\infty$, the locations of the minima of $-L(u, v) / T$ converge in probability to the minima of $E[-L(u, v) / T]$. These minimia define the set $A_{p}^{c}$; hence we need only show that $A_{p}^{c}=D^{c}$. It is straightforward to show that the limit of $E[-L(u, v) / T]$ as $T$ $\rightarrow \infty$ is zero at the $(u, v)$ contained in $D^{c}$, but is nonzero elsewhere. Since the limit of $E[-L(u, v) / T]$ as $T \rightarrow \infty$ is nonnegative, it follows that $A_{p}^{c}=D^{c}$, which proves the result (b).

It follows that wind vector estimation is therefore identifiable to the multimember set $D^{c}$. The wind estimate will be uniquely identifiable (i.e., to a single $(u, v)$ estimate) if and only if $D^{c}$ contains a single member (see Section II).

\section{ConClusions}

In this paper we have considered the identifiability of wind vectors estimated from wind scatterometer measurements of $\sigma^{\circ}$. We have given an estimation of theoretical proof that wind vectors are set-wise identifiable from wind scatterometer measurements. Our results show that due to the nature of the geophysical model function $\mathfrak{T}$ relating the vector wind and the radar backscatter, there will be a set of wind vectors which result from point-wise wind estimation, rather than from a single estimate. In the limit, this set is unique and the vector estimates are consistent. Within this set a selection of a single wind vector cannot be made from the measurements taken at a single sample point. To obtain a single vector estimate, additional constraints such as continuity considerations with nearby measurements must be imposed. This is the first time, to the authors' knowledge, that an estimation-theoretical explanation has been given to support the traditional intuitive approach to wind estimation in which all of the wind vectors corresponding to the local minima of the objective are retained and used in the second, post-estimation processing step known as dealiasing.

While we have shown set-wise identifiability of wind vectors estimated on a point-wise basis, the issue of dealiasing remains. Current approaches to dealiasing are based on various, often ad hoc, methods of selecting single vectors from the noisy ambiguity sets based on comparisons with adjacent noisy ambiguity sets. A theoretical analysis is difficult. We are currently examining techniques for estimating the entire wind field over the observation swath. This fundamentally new approach avoids the need for dealiasing [9]-[11].

\section{REFERENCES}

[1] E. M. Bracalente, D. H. Boggs, W. L. Grantham, and J. L. Sweet, "The SASS ${ }^{1}$ scattering coefficient $\sigma^{\circ}$ algorithm," IEEE J. Oceanic Eng., vol. OE-5, pp. 145-154, Apr. 1980.

[2] C. Chi, D. G. Long, and F. K. Li, "Radar backscatter measurement accuracies using digital Doppler processors in spaceborne scatterometers," IEEE Trans, Geosci. Remote Sensing, vol. GE-24, pp. 426-437, May 1986.

[3] C.-Y. Chi and F. K. Li, "A comparative study of several wind estimation algorithms for spaceborne scatterometers," IEEE Trans. Geosci. Remote Sensing, vol. 26, pp. 115-121, 1988.

[4] J. L. Doob, "Probability and statistics," Amer. Math. Soc. Trans., vol. 36, pp. 759-775, 1934

[5] R. E. Fischer, "Standard deviation of scatterometer measurements from space,'" IEEE Trans. Geosci. Electron., vol. GE-10, pp. 106-113, Mar. 1972.

[6] J. W. Johnson, L. A. Williams, Jr., E. M. Bracalente, F. B Beck, and W. L. Grantham, "Seasat-A satellite scatterometer instrument evaluation,"' IEEE J. Oceanic Eng., vol. OE-5, pp. 138-144, Apr. 1980

[7] F. Li, P. Callalhan, M. Freilich, D. Long, and C. Winn, "NASA scatterometer for NROSS - a system for global oceanic wind measurement,"' in Proc. Int. Geosci. Remote Sensing Symp., 1984, pp. 777-780.

[8] L. Ljung, System Identification: Theory for the User. Englewood Cliffs, NJ: Prentice-Hall, 1987.

[9] D. G. Long and J. M. Mendel, "Model-based estimation of wind fields over the ocean from wind scatterometer measurements, part I: Development of the wind field model," IEEE Trans. Geosci. Remote Sensing, vol. 28, pp. 349-360, May 1990.

[10] D. G. Long and J. M. Mendel, "Model-based estimation of wind fields over the ocean from wind scatterometer measurements, part II: Model parameter estimation,' IEEE Trans. Geosci. Remote Sensing, vol. 28, pp. 361-373, May 1990.

[11] D. G. Long, "Model-based estimation of wind fields over the oceans from wind scatterometer measurements," Ph.D. diss., Univ. Southern California, Los Angeles, 1989.

[12] J. M. Mendel, Lessons in Digital Estimation Theory. Englewood Cliffs, NJ: Prentice-Hall, 1987.

[13] M. A. Donelan and W. J. Pierson, "Radar scattering and equilibrium ranges in wind-generated waves with application to scatterometry," J. Geophys. Res., vol. 92, pp. 4971-5029, 1987.

[14] W. J. Plant, "A two-scale model of short wind-generated waves and scatterometry," J. Geophys. Res., vol. 91, pp. $10735-$ $10749,1986$.

[15] L. D. Schroeder et al., "The relationship between the wind vector and the normalized radar cross section used to derive Seasat-A satellite scatterometer winds," $J$. Geophys. Res., vol. 87, pp. 3318-3336, 1982.

[16] L. C. Schroeder et al., "Removal of ambiguous wind directions for a $K u$-band wind scatterometer using three different azimuth angles,"'IEEE Trans. Geosci. Remote Sensing, vol. GE-23, pp. 91-100, Mar. 1985.

[17] S. J. Shaffer, R. S. Dunbar, S. V. Hsiao, and D. G. Long, "A median-filter-based ambiguity removal algorithm for NSCAT,' IEEE Trans. Geosci. Remote Sensing, vol. 29, pp. 167-174, Jan. 1991.

[18] T. Soderstrom and P. Stoica, System Identification. New York: Prentice-Hall Int., 1989

[19] F. Ulaby, R. Moore, and A. Fung, Microwave Remote SensingActive and Passive. Reading, MA: Addison-Wesley, 1981.

[20] F. J. Wentz, S. Peteherych, and L. A. Thomas, "A model function for ocean radar cross-sections at $14.6 \mathrm{GHz}$, " $J$, Geophys. Res., vol. 89, pp. 3689-3704, 1984.

[21] M. G. Wurtele et al., "Wind direction alias removal studies of Seasat scattermeter-derived wind fields," J. Geophys. Res., vol. 87, no. C5, pp. 3365-3377, 1982.

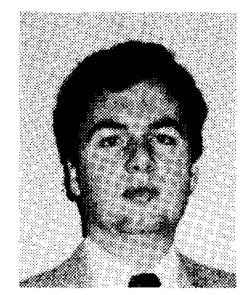

David G. Long (S'80-M'82) received the B.S. and $M . S$. degrees in electrical engineering from Brigham Young University, Provo, UT, in 1982 and 1983, respectively, and the Ph.D. degree in electrical engineering from the University of Southern California, Los Angeles, in 1989.

He has been with the Jet Propulsion Laboratory, Pasadena, CA, as a Radar Systems Engineer since 1983. He has been the Project Engineer for the NASA Scatterometer (NSCAT) project and the Experiment Manager for the Eos SCANSCAT. NSCAT is a spaceborne scatterometer designed to measure 
oceanic winds from space. NSCAT is planned for flight in 1995 aboard the Japanese Advanced Earth Observing System (ADEos). SCANSCAT is a developmental scatterometer being studied for flight on NASA's Earth Observing System (Eos) in the late 1990's. He was responsible for the high level design, analysis, and technical management of these projects to insure that the projects meet all mission requirements. His responsibilities included overseeing instrument design and fabrication, algorithm, and coding for the ground processing system, mission operations, and data analysis. His technical responsibilities included system performance analysis, high-level design, development and maintenance of system requirements, and supervision of the Systems Engineering staff. He is an Assistant Professor in the Electrical and Computer Engineering Department at Brigham Young University. His research interests include computer graphics, speech and signal processing, estimation theory, radar, and mesoscale atmosphere dynamics.

Dr. Long has received the NASA Award of Achievement several times.

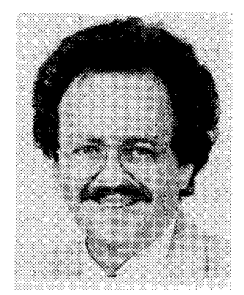

Jerry M. Mendel (S'59-M'61-SM'72-F'78) received the B.S. degree in mechanical engineering and the M.S. and Ph.D. degrees in electrical engineering from the Polytechnic Institute of Brooklyn, Brooklyn, NY, in 1959, 1960 , and 1963 , respectively.

His experience has included teaching courses in electrical engineering at the Polytechnic Institute of Brooklyn from 1960 to 1963, and has also included various consulting positions. From July 1963 to January 1974 he was with the McDonnell-Douglas Astronautics Company. Currently, he is Professor and Chairman of Electrical Engineering-Systems at the University of Southern California, Los Angeles. He teaches courses in estimation theory and seismic data processing for oil exploration, and was Director of the USC Geo-Signal Processing Program (1980-1983). He has published over 230 technical papers and is the author of the monographs: Maximum-Likelihood Deconvolution: A Journey into Model-Based Signal Processing (Springer-Verlag, 1990) and Optimal Seismic Deconvolution: An Estimation-Based Approach (Academic, 1983); the texts: Lessons in Digital Estimation Theory (Prentice-Hall, 1987) and Discrete Techniques of Parameter Estimation: The Equation Error Formulation (Dekker, 1973), and is co-editor (with K. S. Fu) of Adaptive, Learning and Pattern Recognition Systems (Academic, 1970). He is also author of the IEEE Individual Learning Program, Kalman Filtering, and Other Digital Estimation Techniques. He served as Editor of the IEEE Control Systems Society's IEEE TRANSACTIONS ON Automatic Control, for which he presently serves as an Associate Editor-at-Large, and is on the Editorial Board of the IEEE PROCEEDINGS.

Dr. Mendel is a member of the Society of Exploration Geophysicists, the European Association for Signal Processing, Tau Beta Pi, and Pi Tau Sigma, and is a registered Professional Control Systems Engineer in California. He was President of the IEEE Control Systems Society in 1986. He received the SEG 1976 Outstanding Presentation Award for a paper on the application of Kalman filtering to deconvolution, the 1983 Best Transactions Paper Award for a paper on maximum-likelihood deconvolution in the IEEE TRANSACTIONS ON Geoscience and Remote Sensing, a Phi Kappa Phi book award for his research monograph on seismic deconvolution, a 1985 Burlington Northern Faculty Achievement Award, and a 1984 IEEE Centennial Medal. 